\title{
The challenge of exploring organ function: knowledge is not care, but care needs knowledge!
}

\author{
Jean Paul Viale ${ }^{1} \cdot$ Karim Bendjelid ${ }^{2}$
}

Received: 8 December 2017 / Accepted: 10 December 2017 / Published online: 15 December 2017

๑) Springer Science+Business Media B.V., part of Springer Nature 2017

The medical strategies of various diseases are not a straightforward process but are rather the result of complex development. These strategies are generally described as a backand-forth approach between the basic knowledge of disease pathophysiology and observations of symptoms. Results of clinical investigations or monitoring tools are often surrogates of collection of symptom, and therefore, this approach is renewed when an innovative tool becomes available. The respective role of each component, i.e., the basic knowledge, the new technique, and the clinical approach, varies according to the pathology or organ involved in the considered illness, but the rationale stays the same. However, this simplified pattern of medical approach does not fully describe the actual way of medical thinking. Indeed, this later is made more complex as the path of thinking from basic knowledge to symptom is not the same that the one required to discover the cause of a symptom. In other words, the pathways of the mind from basic knowledge to symptom and its reverse are not the same. In the present issue, the review article from Dr Sakka is an interesting opportunity to illustrate this huge difficulty [1].

The present author presents the current position of the use of indocyanine green (ICG) in assessing liver perfusion and function. This molecule has a twofold property that makes it of interest in the liver exploration. First, the transcutaneous spectrophotometric determination of ICG allows the ICG kinetics to be measured in a non-invasive manner, and it can be performed easily at the bedside. Second, due to the particular pharmacodynamics of ICG, which is exclusively taken up by the liver and lacks entero-hepatic recirculation, its elimination rate is exclusively dependent on the liver. As all molecule cleared by the liver, its elimination depends on

\footnotetext{
Jean Paul Viale

jean-paul.viale@univ-lyon1.fr

1 Université de Lyon, 69008 Lyon, France

2 Intensive Care Service, Geneva University Hospitals, 1205 Geneva, Switzerland
}

the liver blood flow and extraction rate, this later being very high in physiological conditions, the elimination is mainly dependent on the liver blood flow.

As the factors leading to change in ICG kinetics are clearly known, it becomes reasonably easy to anticipate the effect of a variation in any of them. For example, a decrease in liver blood flow is expected to decrease ICG elimination and vice versa. If the blood flow remains stable, a change in extraction rate, which is assumed to be equivalent to liver function, leads to a change in the same direction in ICG kinetics. This deduction is a forward way of thinking, leading from physiological knowledge to the clinical effect. Conversely, the clinician faces the opposite position where he knows the result of ICG kinetics and must infer the factor or factors involved in the observed change. ICG kinetics is a warning signal arising from a systematic assessment of liver function or the result of a targeted investigation to quantify a liver injury. Regardless of the demand, the clinician faces a backward process of reasoning, which is far more challenging than the forward process is.

Taking once more the example of ICG kinetics given by Dr Sakka, when interpreting a decrease in an ICG kinetic parameter, the clinician must consider the patient's context to differentiate among the main factors involved, namely the perfusion or the function of the liver. Therefore, the conclusion relies not on a clear-cut relationship but on a more complex procedure that weighs the probabilities of several hypotheses. The issue could be further confused, as several factors may interact in an opposite direction and an additional pathway of ICG elimination may arise in a different disease. For example, Dr Sakka quoted a paper by Stehr et al. [2], who observed stable ICG kinetics in an animal model of sepsis, whereas ICG green biliary excretion decreased as expected in severe sepsis. This observation was ascribed to a temporary ICG redistribution and/or a decrease in the ICG binding protein. Obviously, the clinical relevance of ICG kinetics assessment depends on the setting in which it occurs and on the skills and knowledge of the clinician. 
Some other widely used clinical monitors are not as challenging. The end tidal partial pressure of carbon dioxide (PETCO2) is extensively used in the operating room and ICU and is usually thought to be a warning signal. Therefore, it is monitored on a continuous basis. A change in PETCO2 is a signal that something is occurring, but the "something" is not directly known. Knowledge of the pathways of carbon dioxide from cell production to lung elimination suggests that this change could be the result of changes in cell metabolism (sepsis, hyperthermia), cardiac output, alveolar ventilation, or lung dead space size, which represent wide varieties of events pertaining to metabolism, circulation and ventilation. The consideration of context associated with plain reasoning is usually more than sufficient to identify the correct explanation and, therefore, the adapted management.

Dynamic indicators arising from cardiopulmonary interactions in ventilated patients are another example of the way clinicians manage the medical use of new available indices. Stroke volume variations (SVV) during mechanical ventilation are a well-known heart-lung interaction effect of the ventilation-induced intrathoracic pressure swing. The mechanism of SVV is extensively described, and mainly refers to the phasic change in the gradient of pressure driving the venous blood return as a consequence of the change in intrathoracic pressure [3, 4]. Therefore, any observation of a significant oscillation in stroke volume or its surrogates, such as pulse pressure, with mechanical breathing indicates that the patient will respond with an increase in cardiac output to a volume loading. This is the classical intrinsic adaptive regulation of cardiac output, allowing the heart to increase its outflow when facing an increased inflow, according to the well-known Frank Starling curve. Therefore when fluctuations are encountered, this is indicative that the patient volemia is in an adaptive zone. From the other side, i.e., the clinician's point of view, the observation of a significant oscillation in SVV, or its surrogates, is usually understood as a necessity to perform volume loading, until the fluctuation reaches a low threshold value, usually under $13 \%[5,6]$. This discrepancy between the physiological meaning of an observed fluctuation on the one hand and the conclusion drawn on the other is amazing, and several explanations may be given. The first explanation, to stick with the physiological understanding, is that the normal functioning of a left ventricle is just beneath the flat part of the Frank Starling curve, where large variations in left ventricle volume induce small changes in stroke volume. This assertion is awaiting validation. The second explanation is more subjective and relates to the scarcity of tools available to evaluate the volemia of a patient. However crucial it is to adapt blood volume in circulatory disorders, this information is among the most difficult indication to acquire in clinical settings. Therefore, the availability of any signal relative to these data is welcome. The primary aim of physicians being care, this index has played a role as an indicator of management in prompting the caregiver to perform volume loading. Notably, the observation of a fluctuation in circulatory index has been qualified a "preload dependency", which is a literal interpretation. However, owing to its negative connotation, the swing in the index suggests the need to achieve independency. One could question the behaviour of caregivers if these indices were qualified as "adaptive".

In conclusion, thinking about the role of new tools in our medical environment goes far beyond the initial reflection of ICG kinetics, but it seems a worthy endeavour to pause and discern our unrevealed or occult methods for the use of newly available tools. Indeed, as suggested above, interpreting the data from our diagnostic tools may follow diverse paths, from a purely physiological rationale (PETCO2) to a choice between weighted hypotheses (ICG kinetics) and to an analysis that is guided by the need to have tools to manage difficult issues (SVV and surrogates).

\section{References}

1. Sakka SG. Assessment of liver perfusion and function by indocyanine green in the perioperative setting and in critically ill patients. J Clin Monit Comput. 2017. https://doi.org/10.1007/ s10877-017-0073-4.

2. Stehr A, Ploner F, Traeger K, Theisen M, Zuelke C, Radermacher $\mathrm{P}$, et al. Plasma disappearance of indocyanine green: a marker for excretory liver function? Intensive Care Med. 2005;31:1719-22.

3. Bendjelid K, Romand JA. Fluid responsiveness in mechanically ventilated patients: a review of indices used in intensive care. Intensive Care Med. 2003;29:352-60.

4. Michard F. Changes in arterial pressure during mechanical ventilation. Anesthesiology. 2005;103:419-28.

5. Scheeren TWL, Wiesenack C, Gerlach H, Marx G. Goal-directed intraoperative fluid therapy guided by stroke volume and its variation in high-risk surgical patients: a prospective randomized multicentre study. J Clin Monit Comput. 2013;27:225-33.

6. Benes J, Chytra I, Altmann P, Hluchy M, Kasal E, Svitak R, et al. Intraoperative fluid optimization using stroke volume variation in high risk surgical patients: results of prospective randomized study. Crit Care. 2010;14:R118. 